\title{
Determination of Ketoprofen Enantiomers in Plasma by Solid Phase Extraction and Column Switching High Performance Liquid Chromatography
}

\author{
Gen Tamai, Masami Edani and Hideo ImaI \\ Faculty of Pharmaceutical Sciences, Fukuyama University, Higashimuracho, Fukuyama 729-02, Japan
}

\begin{abstract}
Protein-coated cyanopropyl silica gel packed in a precolumn was successfully used as an adsorbent for ketoprofen in blood plasma. After column switching, ketoprofen collected in the precolumn was transferred into an ovomucoid column, and the chiral separation was carried out by a mobile phase of $0.1 \mathrm{M}$ phosphate solution (pH 3.2) containing $20 \%$ methanol. The separation factor was 1.32 , and the resolution was 1.30 . The recovery by direct injection of plasma samples was approximately $100 \%$, with a relative standard deviation of about $5 \%$ between days $(n=6)$. The calibration graph of each enantiomer was a straight line up to $1 \mathrm{nmol}$; each detection limit was 5 pmol $(S / N=5)$.
\end{abstract}

Keywords Column switching high performance liquid chromatography, solid phase extraction, ketoprofen enantiomer, blood plasma sample, direct injection, on-line deproteinization

The column switching high performance liquid chromatography (HPLC) coupled with solid phase extraction in a precolumn was a useful technique for analyses of drugs or biological constituents in complex matrices. ${ }^{1,2}$ Recently, we employed this technique for chiral separation and determination of propranolol enantiomers in blood or tissues. ${ }^{3}$ A chiral stationary phase using a biopolymer such as bovine serum albumin $(B S A)^{4}, \alpha_{1}$-acid glycoprotein ${ }^{5}$ or ovomucoid $\left(\mathrm{OVM}^{6}{ }^{6}\right.$ was recommended for a sample in aqueous solution. An aqueous mobile phase containing an organic solvent could be used for the chiral separation. It is essential that the analyte collected in a precolumn be eluted and transferred perfectly into the chiral column and separated by the same mobile phase. Choice of a precolumn packing was also necessary in order to ensure the quantitative recovery and perfect elution.

Ketoprofen (KP), $R, S-2-(3-$ benzoylphenyl)propionic acid, is a clinically important non-steroidal antiinflammatory drug; ${ }^{7}$ it possesses a chiral center in the propionic acid moiety. Commercially, KP can be obtained in the racemic form. An in vitro experiment proved that the $S(+)$ enantiomer exhibited pharmacological effects, but $R(-)$ was inactive. An in vivo experiment showed that the chiral inversion took place from $R(-)$ to $S(+)$, the degree of inversion being varied from one animal species to another. ${ }^{8}$ Therefore, the stereoselective analysis attracts attention in relation to the pharmacokinetic studies.

We developed a method of column switching HPLC with direct injection of plasma samples for chiral separation of KP by using a protein-coated cyanopropyl silica precolumn for solid phase extraction and an OVM-bonded silica column for chiral separation. We applied this method to study the time course of elimination of KP enantiomers in rat plasma. The details are presented in this paper.

\section{Experimental}

\section{Materials}

Racemic KP was obtained from Sigma (St. Louis, MO, USA). Human plasma was purchased from Cappel (West Chester, PA, USA). All other chemicals and solvents were of analytical reagent grade, and were used without further purification.

\section{HPLC}

A short precolumn $(1 \mathrm{~cm} \times 4 \mathrm{~mm}$ i.d. $)$ was packed with cyanopropyl silica gel (Nucleosil 5CN, MachereyNagel, Düren, Germany). The external surface of the porous gel was coated with plasma proteins by the same procedure as reported in the previous paper. 9 A guard column ( $1 \mathrm{~cm} \times 4 \mathrm{~mm}$ i.d.) packed with aminopropyl silica gel (Develosil $\mathrm{NH}_{2}-5$, particle size $5 \mu \mathrm{m}$, Nomura Chemical Co., Ltd., Seto, Japan) was attached to the precolumn in order to protect the precolumn from interfering endogenous constituents in blood. An OVMbonded silica column (Ultron ES-OVM, $15 \mathrm{~cm} \times 4.6 \mathrm{~mm}$ i.d., Shinwa Kako Co., Kyoto, Japan) was used for the chiral separation. A guard column (Ultron ES-OVM $\mathrm{G}, 1 \mathrm{~cm} \times 4 \mathrm{~mm}$ i.d., Shinwa Kako Co.) was attached to 
the OVM column.

A Waters Model 501A (Waters, Division of Millipore, Bedford, MA, USA) was used for the HPLC pump. The flow rate was $0.8 \mathrm{ml} / \mathrm{min}$ throughout the experiment. Detection was carried out at $265 \mathrm{~nm}$ with a Waters Model 484 Tunable Absorbance Detector. The column switching device and other pieces of apparatus were the same as reported in the previous paper. ${ }^{10,11}$

\section{Results and Discussion}

\section{Enantiomer resolution}

The chiral resolution of KP in an OVM column was remarkably influenced by electrolyte concentration, $\mathrm{pH}$ and organic modifiers in the mobile phase.

In the absence of electrolyte, KP was not eluted over $1 \mathrm{~h}$ even by the presence of $15 \%$ ethanol or $20 \%$ methanol. The elution and enantiomer resolution were dramatically improved by the addition of electrolyte (phosphate, 50-200 mM). Figure 1(A) shows the effect of phosphate concentration on the capacity factor, $k^{\prime}$. In this case, phosphate concentration as high as $50 \mathrm{mM}$ was preferable.

The value of the separation factor, $\alpha$, increased gradually with decrease of $\mathrm{pH}$ from 6.5 to 3.0 in solution of $50 \mathrm{mM}$ phosphate containing $10 \%$ ethanol. The value of $k^{\prime}$ showed a maximum at about $\mathrm{pH} 4.0$ (Fig. 1(B)). It is noteworthy that the isoelectric point of $\mathrm{OVM}$ is 4.3 and $\mathrm{p} K_{\mathrm{a}}$ of $\mathrm{KP}$ is 3.9. Therefore, it seems

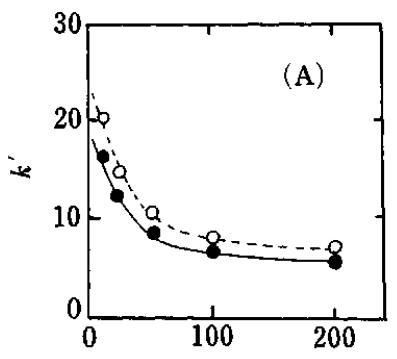

Phosphate concentration $/ \mathrm{mM}$

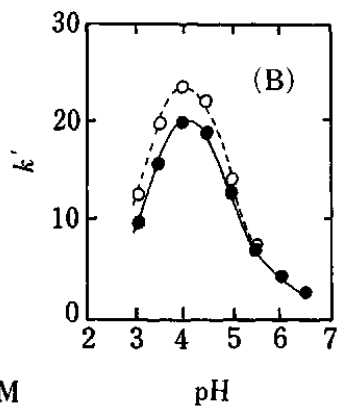

Fig. 1 Effect of phosphate concentration (A) and $\mathrm{pH}$ (B) on $k^{\prime}$ value. $S(+) \mathrm{KP} ; \mathrm{O}, R(-) \mathrm{KP}$.

Table 1 Effect of organic modifiers

\begin{tabular}{llrc}
\hline \multicolumn{1}{c}{ Organic modifier } & & \multicolumn{1}{c}{$k^{\mathrm{b}}$} & \multicolumn{1}{c}{$\alpha$} \\
\hline Methanol & $(15-25 \%)$ & $13.4-4.0$ & $1.4-1.2$ \\
Ethanol & $(10-15 \%)$ & $12.5-6.1$ & $1.3-1.1$ \\
1-Propanol & $(5-15 \%)$ & $9.4-2.1$ & $1.1-1.0$ \\
2-Propanol & $(7.5-15 \%)$ & $9.6-3.0$ & $1.1-1.0$ \\
Acetonitrile & $(10-15 \%)$ & $8.6-2.5$ & $1.1-1.0$ \\
Tetrahydrofuran & $(5-10 \%)$ & $7.7-3.8$ & $1.1-1.0$ \\
\hline
\end{tabular}

a. Addition to $50 \mathrm{mM}$ phosphate solution (pH 3.2).

b. $S(+)$ KP. that the $k^{\prime}$ value increases at about $\mathrm{pH} 4.0$ owing to the interaction between $\mathrm{KP}$ anion and positively charged OVM protein.

When the carbon number of primary normal alcohols was increased from 1 to 3 , both $k^{\prime}$ and $\alpha$ were decreased. Among the organic modifiers investigated, $20 \%$ methanol was most preferable, as shown in Table 1.

The value of $k^{\prime}$ decreased with increase of temperature from $15^{\circ}$ to $40^{\circ} \mathrm{C}$. In this connection, $20^{\circ} \mathrm{C}$ was most preferable.

When a mobile phase of $0.1 \mathrm{M}$ phosphate solution (pH 3.2) containing 20\% methanol was used, the retention time was $9.6 \mathrm{~min}$ for $S(+)$ and $12.0 \mathrm{~min}$ for $R(-)$, and chiral separation was satisfactory $(\alpha=1.32$ and $R_{\mathrm{s}}=1.30$ ) under the column temperature at $20^{\circ} \mathrm{C}$. The free energy difference in the chiral resolution was estimated to be as small as $-163 \mathrm{cal} / \mathrm{mol}$ from the separation factor.

\section{Solid phase extraction}

$\mathrm{C}-18$ bonded silica gel strongly adsorbed KP, so that the elution was difficult by using the mobile phase suitable for the chiral separation. C-8 bonded silica gel, a hydrophobically interacting adsorbent gel (Butyl Toyopearl $650-\mathrm{M}$ ) or an anion exchange resin showed low recovery or inadequate elution for KP. Only mildly adsorbing cyanopropyl silica was suitable to elute out with an eluant containing a low concentration of organic modifiers.

Figure 2(A) shows the chromatogram of standard aqueous KP injected on a column $(1 \mathrm{~cm} \times 4 \mathrm{~mm}$ i.d. $)$ packed with protein-coated cyanopropyl silica gel and eluted by $0.1 \mathrm{M}$ phosphate solution ( $\mathrm{pH}$ 3.2). When the same quantity of KP spiked in blood plasma was injected, the KP peak was slightly broadened (Fig. 2(B)). The broadening may be caused by the $\mathrm{pH}$ shift of the eluant due to plasma proteins. When the plasma sample was acidified (pH 3-4) with the same volume of $0.1 \mathrm{M}$ phosphoric acid, peak broadening was no longer observed (Fig. 2(C)).

Plasma proteins in the sample were not adsorbed on

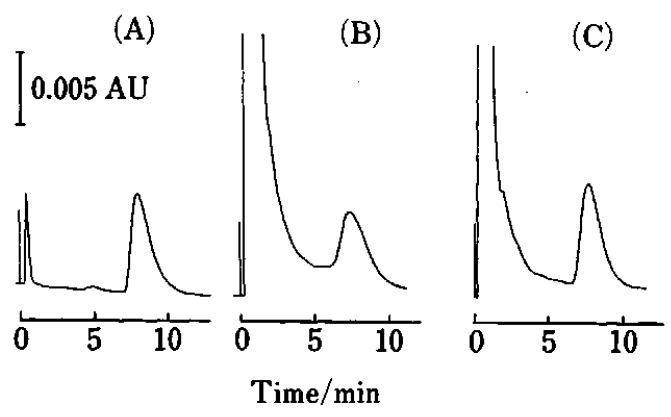

Fig. 2 Elution profile of $2 \mathrm{nmol} \mathrm{KP}$ from precolumn. Samples: (A) standard aqueous sample, (B) standard spiked in plasma and (C) the plasma sample acidified with phosphoric acid. 
Table 2 Recovery of KP enantiomers in plasma

\begin{tabular}{ccr}
\hline \multirow{2}{*}{ Concentration $/ \mu \mathrm{M}$} & \multicolumn{2}{c}{ Recovery, \% } \\
& $S(+)$ & $R(-)$ \\
\hline 1 & 100.5 & 98.5 \\
10 & 101.1 & 101.3 \\
50 & 99.5 & 97.5 \\
\hline
\end{tabular}

the protein-coated cyanopropyl silica, and were eluted at the void volume. The recovery of the eluted proteins from the plasma sample was estimated to be $95-102 \%$ by the Commassie Brilliant Blue method. Plasma proteins coated on the gel were not eluted until $40 \%$ methanol. When the mobile phase of $0.1 \mathrm{M}$ phosphate solution (pH 3.2) containing $20 \%$ methanol was used, the recovery of KP in plasma samples was approximately $100 \%$ (Table 2 ), regardless of the high protein binding ratio of KP. The protein binding ratio is reported to be over $90 \%$ at the therapeutic concentration of $5-12 \mu \mathrm{g} / \mathrm{ml} .^{12}$ Deterioration of the precolumn by some endogenous constituents was avoided by a short guard column $(1 \mathrm{~cm} \times 4 \mathrm{~mm}$ i.d.) packed with aminopropyl silica gel.

\section{Column switching HPLC}

A mobile phase of $0.1 \mathrm{M}$ phosphate solution ( $\mathrm{pH}$ 3.2) was delivered to the precolumn. A plasma sample $(20 \mu \mathrm{l})$ diluted and acidified by the same volume of $0.1 \mathrm{M}$ phosphoric acid was injected onto the precolumn at room temperature. Proteins and hydrophilic components in plasma were eluted and discarded. In 5 min after injection, the precolumn was connected to the OVM column in stream. The OVM column was previously equilibrated with $0.1 \mathrm{M}$ phosphate solution (pH 3.2) containing $20 \%$ methanol, and controlled the temperature at $20^{\circ} \mathrm{C}$. Then, by a mobile phase of $0.1 \mathrm{M}$ phosphate solution (pH 3.2) containing $20 \%$ methanol, KP collected in the precolumn was eluted, transferred into the OVM column, and KP enantiomers were separated in the OVM column by the same mobile phase. KP enantiomers were detected spectrophotometrically at $265 \mathrm{~nm}$. During the separation on the OVM column, the precolumn was rinsed with $0.1 \mathrm{M}$ phosphate solution ( $\mathrm{pH} 7.0$ ) containing $20 \%$ acetonitrile for $3 \mathrm{~min}$, water for $1 \mathrm{~min}$, and then absolute methanol for $3 \mathrm{~min}$.

\section{Analysis of KP in blood plasma sample}

The typical elution profiles of KP in a plasma sample at the initial use of an OVM column and that after 200 times injection are shown in Figs. 3(A) and 3(B), respectively. The resolution was still good after 200 times use, though the shapes of artifact peaks were changed. The calibration graph showed a straight line in the concentration range from $5 \mathrm{pmol}$ to $1 \mathrm{nmol}$ of each enantiomers. The recovery data were estimated by comparing the elution peak height of an aqueous



Fig. 3 Elution profile of KP enantiomers in plasma obtained with (A) new OVM column and (B) the column after 200 times injection. Sample: human plasma $(20 \mu \mathrm{l})$ spiked with 100 pmol racemic $K P$ after acidification with phosphoric acid. Eluent: $0.1 \mathrm{M}$ phosphate solution ( $\mathrm{pH} 3.2$ ) containing $20 \%$ methanol.

Table 3 Reproducibility by the present method

\begin{tabular}{llcc}
\hline & & \multicolumn{2}{c}{$\mathrm{RSD}, \%$} \\
& & $S(+)$ & $R(-)$ \\
\hline Within day & $(n=10)$ & 1.5 & 1.7 \\
Between days & $(n=6)$ & 4.5 & 5.2 \\
\hline
\end{tabular}

Sample: $20 \mu$ l of plasma spiked with $100 \mathrm{pmol}$ of racemic ketoprofen.

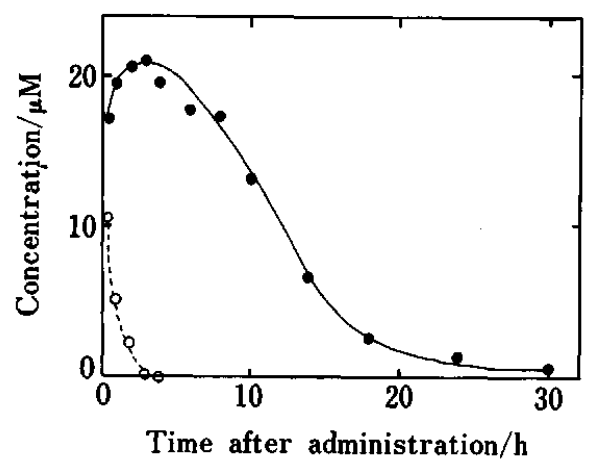

Fig. 4 Concentration of KP enantiomers in blood plasma of a rat after oral administration. $-S(+) \mathrm{KP} ; \mathrm{O}, R(-) \mathrm{KP}$.

standard sample and plasma sample spiked with KP (Table 2). The reproducibility was expressed by the relative standard deviation (RSD) of successive injections (Table 3). The OVM column was rinsed with $30 \%$ aqueous 2-propanol solution every day after use; thus, the OVM column could be used over 200 times for plasma samples.

Racemic KP was dissolved in $0.04 \mathrm{M}$ phosphate buffer ( $\mathrm{pH} 7.4$ ), and administered orally to a Wister rat (dose, $2.5 \mathrm{mg} / \mathrm{kg}$ ). The plasma concentration of KP 
enantiomers after administration is shown in Fig. 4. $R(-) \mathrm{KP}$ eliminated fairly quickly, and in $3 \mathrm{~h}$ it mostly disappeared, while $S(+)$ KP showed a maximum blood concentration at about $3 \mathrm{~h}$ after administration. A coupled effect of the metabolic chiral inversion and elimination pathway seems to take place in this case.

\section{References}

1. H. Imai, T. Masujima, I. Morita-Wada and G. Tamai, Anal. Sci., 5, 389 (1989).

2. R. D. McDowall, J. Chromatogr., 492, 3 (1989).

3. G. Tamai, M. Edani and H. Imai, Biomed. Chromatogr., 4, 157 (1990).

4. R. A. Thompson, S. Andersson and S. Allenmark, $J$. Chromatogr., 465, 263 (1989).

5. J. Hermansson, J. Chromatogr., 269, 71 (1983).
6. T. Miwa, M. Ichikawa, M. Tsuno, T. Hattori, T. Miyakawa, M. Kayano and Y. Miyake, Chem. Pharm. Bull., 35, 682 (1987).

7. R. N. Brodgen, T. M. Speight and G. S. Avery, Drugs, 8, 168 (1974).

8. A. J. Hutt and J. Caldwell, J. Pharm. Pharmacol, 35, 693 (1983).

9. H. Yoshida, I. Morita, G. Tamai, T. Masujima, T. Tsuru, N. Takai and H. Imai, Chromatographia, 19, 466 (1984).

10. G. Tamai, H. Yoshida and H. Imai, J. Chromatogr., 423, $147,155,163$ (1987).

11. H. Imai and G. Tamai, Biomed. Chromatogr., 3, 192 (1989).

12. R. L. Williams, R. A. Upton, J. N. Buskin and R. M. Jones, Clin. Pharmacol. Ther., 30, 226 (1981).

(Received August 13, 1990) (Accepted October 25, 1990) 